\title{
The Role of Fish Oil in Inflammatory Eye Diseases
}

\author{
Hong Wang Bruce P. Daggy \\ Research and Development, Shaklee Corporation, Pleasanton, CA, USA
}

\begin{abstract}
What Is It about?
This article reviews the evidence that fish oil can help manage two common inflammatory eye diseases - dry eye disease and age-related macular degeneration. It also describes mechanisms, including conversion of long-chain omega-3 fatty acids into some recently discovered anti-inflammatory mediators, that may explain the beneficial effects of fish oil on these diseases. It attempts to explain discrepancies in the clinical data and makes a recommendation as to the effective dose.
\end{abstract}

\section{Keywords}

Fish oil · Omega-3 fatty acids · Anti-inflammation · Dry eye disease $\cdot$ Age-related macular degeneration · Omega-3 Index

\begin{abstract} eases.

Consumption of fish oil is associated with reduced morbidity and mortality of cardiovascular diseases and also reduces the severity of many other inflammatory diseases and autoimmune disorders. The beneficial effects are attributed to the anti-inflammatory effects of the omega-3 polyunsaturated fatty acids, eicosapentaenoic acid (EPA) and docosahexaenoic acid (DHA), found in fish oils. The mechanism of the anti-inflammatory effects was long thought to be by modulating the production of proinflammatory mediators, including prostaglandins, thromboxanes, and leukotrienes. Recent advances in research into the novel lipid mediators (resolvins, protectins, and maresins) derived from EPA and DHA and their role in the resolution of inflammation have shed new light on the pleiotropic nature of these fatty acids. In this review, we focus on the effects of EPA and DHA from fish oil in the treatment of two common inflammatory eye diseases - dry eye disease and age-related macular degeneration. Evidence from recent studies lends support to a role of fish oil in the treatment of these two eye dis- 


\section{Introduction}

Inflammation is a vital self-defense immunological mechanism to protect organisms from infection, injury, trauma, and other insults. The defense mechanisms against infection can be nonspecific (e.g., leukocytes and macrophages against bacteria) or specific (e.g., antibody-mediated inactivation of inflammatory agents involving dendritic cells, lymphocytes, and plasma cells). Leukocytes migrate from the blood into the affected surrounding tissue, a process promoted by chemoattractants from the site of inflammation and by the release of chemical mediators, which include prostaglandins and leukotrienes. The influx of cells into the site of inflammatory activity and the presence of the inflammatory mediators produced as a result generate the cardinal signs of inflammation: redness, swelling, heat, pain, and loss of function [1]. Since acute inflammation is a protective response to eliminate invading organisms and to permit repair of injured tissue, it is ideally self-limiting and leads to a complete resolution enabling a return to homeostasis [2]. For a successful outcome, the acute inflammatory response must be triggered to bring about recruitment of neutrophils, activation of macrophages, and production of a series of mediators to enable an ultimate resolution of the inflammatory process, triggering events that lead to elimination of invading organisms, tissue regeneration, and wound healing. Failure to come to a resolution may lead to excessive or chronic persistent inflammation, which is an underlying pathology in many diseases, such as cardiovascular diseases, neurodegenerative diseases, and eye diseases.

The key lipid mediators that are generated during inflammation are derived from the fatty acids that are incorporated into cell membrane phospholipids. Dietary lipids have a direct impact on the types and amount of fatty acids incorporated into the cell membranes. Phospholipids of blood cells taken from humans consuming a typical Western diet typically contain $15-20 \%$ of omega- 6 fatty acids as arachidonic acid (ARA), omega- 3 (n-3) fatty acids as $0.5-1 \%$ eicosapentaenoic acid (EPA), and 2-3\% docosahexaenoic acid (DHA) [1]. Eicosanoids are the lipid mediators generated by metabolic pathways from these fatty acids. ARA is usually the major substrate for eicosanoid biosynthesis due to its abundance in the cell membrane. Free ARA cleaved from membrane phospholipids can then convert into different classes of eicosanoids (2-series prostaglandins, thromboxanes, 4-series leukotrienes, hydroxyeicosatetraenoic acids, etc.) dependent on various cellular enzymes (cyclooxygenases [COX], lipoxygenases [LOX], and cytochrome P450 enzymes, etc.). These eicosanoids from ARA are generally proinflammatory and are initiated during the acute phase of inflammation. In both animal and human experiments, incorporation of marine $n-3$ fatty acids, such as EPA and DHA, into the membrane phospholipids of cells involved in inflammation occurs in a time- and dose-dependent fashion and at the expense of ARA [1]. Therefore, it was thought that the beneficial effects of taking fish oil are the result of displacing ARA by EPA and/or DHA, which then lowers the generation of proinflammatory eicosanoids. In addition, EPA and DHA were also shown to inhibit ARA metabolism, including decreasing the gene expression of COX-2 [1, 3], which generates the proinflammatory eicosanoids. Human studies have shown that oral intake of fish oil (EPA + DHA) decreases the production of ARA-derived eicosanoids [1, 4]. Similar to ARA, EPA, a 20-carbon unsaturated fatty acid, is also a substrate for the eicosanoid-producing COXs, LOXs, and cytochrome P450 enzymes. However, due to structural differences, mediators generated from EPA give rise to 3 -series prostaglandins, thromboxanes, and 5-series leukotrienes, which are in general less biologically potent than mediators from ARA [1]. Therefore, replacing ARA by EPA provides at least 2 ways to reduce the inflammatory response. In addition, recent advances in the uncovering of novel classes of mediators of resolution of inflammation showed that resolution is an active programmed response that is turned on during the 




Fig. 1. Pro-resolving endogenous mediators: lipoxin, resolvin, protectin, and maresin.

course of inflammation [2]. These lipid mediators (resolvins, protectins, and maresins) derived from EPA and, for the most part, from DHA shed new light on the anti-inflammatory and pro-resolving activities provided by taking fish oil.

\section{Resolution of Inflammation and Pro-Resolving Mediators Derived from Long-Chain n-3 Fatty Acids}

Based on the review by Buckley et al. [5], the process of resolution of inflammation includes 5 key steps: (1) clearance of the inciting stimuli; (2) catabolism of local survival signals and silencing of intracellular proinflammatory signaling pathways; (3) normalization of chemokine gradients; (4) efferocytosis by tissue and macrophages, and (5) either incorporation of these myeloid cells into the local population or their recirculation via lymph or blood. Lipid mediators, including the eicosanoids and the pro-resolving mediators derived from ARA, EPA, and DHA, represent key signaling molecules in the process which regulates the inflammatory profile and promotes the return of affected tissues to homeostasis.

In fact, it is now known that ARA is also a substrate that can generate lipoxins (LXA4), a class of pro-resolving lipid mediators, via 12/15-LOX due to class switching, a temporal switch in inflammatory exudates during acute inflammation to regulate leukocyte infiltration $[6,7]$. The n-3 fatty acids EPA and DHA are the substrates for the biosynthesis of potent antiinflammatory and pro-resolving endogenous mediators, such as resolvins, protectins, and maresins, via COX-2, 5-LOX, and 12/15-LOX (Fig. 1). These specialized pro-resolving mediators (SPMs) exert diverse biological effects on immune function, including the ability to counterregulate mediators that trigger leukocyte trafficking [5]. Functionally, SPMs enhance bacterial clearance by stimulating mucosal production of bacterial peptides, enhancing bacterial phagocytosis by leukocytes and macrophages, and working synergistically with antibiotics to enhance their therapeutic action. Importantly, although they encourage resolution, they do not compromise host immune competence but can increase survival from 
infection in animals [5]. Therefore, functionally, these SPMs could be called endogenous "healing" molecules and may exert protective effects against inflammatory eye diseases, such as dry eye disease (DED) and age-related macular degeneration (AMD).

A PubMed search was conducted using the terms "fish oils," "dry eye," and "age-related macular degeneration." Of the articles retrieved by this method, we reviewed all publications in English and abstracts of non-English publications, which included articles that described the incidence, pathogenesis, prevention, and treatment of inflammatory eye diseases. Emphasis was placed on articles published since the review on "Omegas and Dry Eye" by Hom et al. [8], but we included earlier articles that provided a more comprehensive understanding of n-3 fatty acids in inflammatory eye diseases, including AMD. We listed clinical trial articles that described the treatment for DED and AMD with a treatment group using only n-3 fatty acids from fish oil, so that the efficacy of the treatment was solely due to the $n-3$ fatty acids and not to a combination with other nutrients or antioxidants. Table 1 provides a brief summary of selected clinical studies using fish oil for these 2 diseases.

\section{Dry Eye Disease}

Based on the Report of the International Dry Eye Work Shop (DEWS) [9, 10], DED is defined as a "multifactorial disease of the tears and ocular surface that results in symptoms of discomfort, visual disturbance, and tear film instability with potential damage to the ocular surface." It is accompanied by increased osmolarity of the tear film and subacute inflammation of the ocular surface. The ocular surface (cornea, conjunctiva, and accessory lacrimal glands), meibomian glands (specific sebaceous glands of the eyelid margin, which produce the outer lipid film of the tear film), the main lacrimal gland, and the innervations between them form a functional unit. Any or all of these structures may be affected in DED. Recent studies have shown that dry eye is an inflammatory disease that has many features in common with autoimmune disease [10]. Stress to the ocular surface (environmental factors, infection, endogenous stress, antigens, and genetic factors) is postulated as the pathogenic triggering mechanism [10]. The meibomian glands, found in the upper and lower eyelids, excrete lipids onto the ocular surface that form the outermost layer of the tear film, lubricating the ocular surface during blinking and protecting it against tear evaporation. Through dysfunction of the meibomian glands, reduced lipid secretion may contribute to tear film instability and entry into the vicious circle of DED. Indeed, meibomian gland dysfunction is the most common cause of evaporative DED [11]. For years, patients with dry eye have used treatments, such as artificial tears, ointments, and punctal plug (tear duct plug), for symptom relief only. Anti-inflammatory drugs, such as steroids or cyclosporine A, can be used but with potential side effects.

A recent double-blind clinical study evaluated the efficacy of oral intake of a combined nutraceutical formulation containing $\mathrm{n}-3$ fatty acids ( $1.27 \mathrm{~g} /$ day) with vitamins and minerals for 3 months targeting meibomian gland dysfunction [12]. The results showed beneficial effects of the treatment with improvement in tear stability and reduction of eyelid inflammation. Indeed, based on a meta-analysis of randomized controlled clinical studies [13], oral n-3 fatty acid supplementation was associated with better tear breakup time and improvement in Schirmer's test (measurement of the secretions of the lacrimal gland) [10]. Oral intake of n-3 fatty acids was also found to have beneficial effects on dry eye in contact lens wearers [14], computer vision syndrome-related dry eye [15], visual display terminal users [16], and rosacea patients having dry eye symptoms [17]. A summary of the clinical studies is given in Table 1a. These clinical studies' results provide some direct evidence to support the observation in the Women's Health Study with 32,470 participants showing that women with higher intake of n-3 fatty acids have a decreased prevalence of dry eye syndrome [18]. 


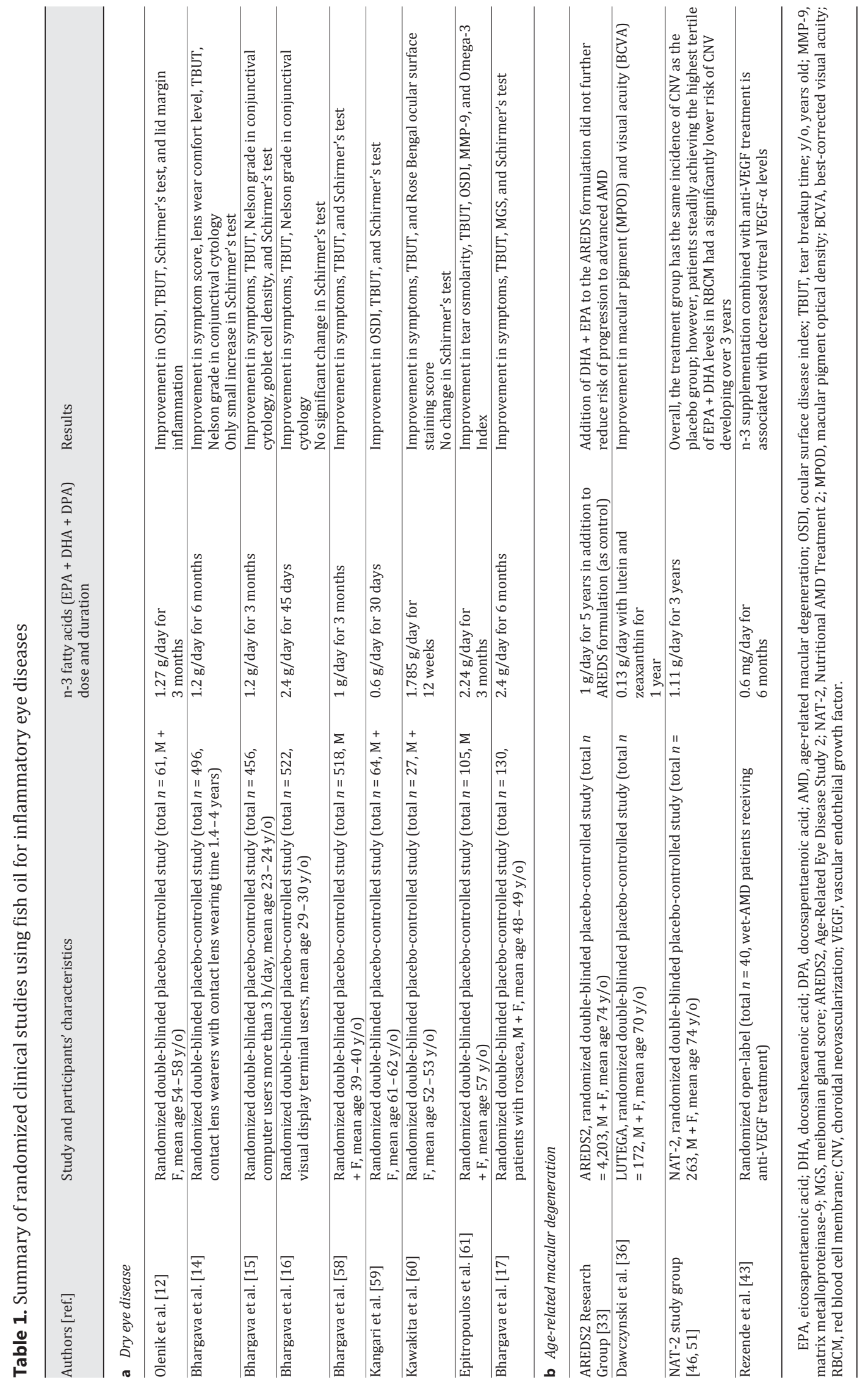


Severe dry eye is one of the complications of patients who undergo corneal surgery. It is estimated that 1.5 million people undergo refractive surgery, such as LASIK (laser in situ keratomileusis) and photorefractive keratectomy, in the United States each year [19]. The cornea is heavily innervated [20]. Dry eye can arise from sensory denervation during the surgery. Based on the novel lipid mediators and their role in resolution of inflammation, DHA was tested by direct application to eyes topically with pigment epithelial-derived growth factor in animal studies of dry eye and corneal nerve regeneration after corneal surgery. The results showed that neuroprotectin D1 (NPD1), a SPM derived from DHA, was detected in the tissue and that DHA enhanced the nerve regeneration effect of pigment epithelial-derived growth factor [19] and reduced the biosynthesis of the proinflammatory mediator leukotriene B4 [21]. Additional study showed that NPD1 directly enhanced corneal nerve regeneration similarly to DHA and pigment epithelial-derived growth factor and had anti-inflammatory effects by reducing neutrophil infiltration [22]. These results further illustrated the healing effects of n-3-derived SPM. Receptors for SPMs (RvE1 and $\left.\mathrm{LXA}_{4}\right)$ were detected in the corneal tissue (epithelium, stromal-endothelial layers, and CD11b+ immune cells [macrophage or dendritic cell]) in mice [23]. Topical application of SPMs (RvE1 and RvD1) and an $\mathrm{LXA}_{4}$ analog to inflamed eyes with suture-induced corneal neovascularization reduced the infiltration of neutrophils and macrophages, proinflammatory cytokines (tissue necrosis factor- $\alpha[$ TNF- $\alpha]$ and IL-1 $\beta$ ), vascular endothelial growth factor (VEGF) expression, and angiogenesis [23]. Currently, there are SPM analogues developed for topical use as a treatment option for ocular surface inflammation and disease [22].

\section{Age-Related Macular Degeneration}

AMD is the degeneration of the retinal macula, the region responsible for central vision. AMD is a leading cause of blindness in the United States and England [24]. Clinical findings associated with AMD include drusen (extracellular deposits of protein-carbohydrate-lipid complexes under the retina) and abnormalities in retinal pigment epithelium, including detachment, geographic atrophy, and choroidal neovascularization (CNV), which may include retinal detachment with hard exudates, subretinal hemorrhages, and scar formation [24]. The prevalence of identifiable drusen increases with age, especially after the sixth decade. Larger and more extensive drusen seem to be associated with the risk of central visual acuity loss. This vision loss can come from the development of CNV, the neovascular form (wet AMD) that causes most of the severe vision loss from AMD, or from nonneovascular lesions, such as geographic atrophy (dry AMD) [24]. Neovascular AMD is a complex disease which may be triggered by aging and environmental stress in an individual with genetic predisposition. The pathophysiological signaling pathways may involve inflammation, oxidative stress, and/or angiogenesis in the retinal pigment epithelium cells and choroidal endothelial cells, leading to vision loss from $\mathrm{CNV}[25,26]$. There is no effective treatment for most cases of AMD; however, a small proportion of patients with well-defined CNV benefit from laser photocoagulation [24]. Recent advances in the development of intravitreal antiangiogenic therapies (anti-VEGF antibody injection) for neovascular AMD have greatly improved clinical outcomes in the last decade; however, vision improvement during the first 2 years was not maintained at 5 years, and monthly treatments were associated with an increased risk of developing geographic atrophy [27]. Currently, treatments remain inadequate for atrophic AMD [26].

Due to the prevalence of the diseases, the National Eye Institute (NEI) of the National Institutes of Health (NIH) sponsored the Age-Related Eye Disease Study (AREDS), a long-term multicenter prospective study of 4,757 people aged 55-80 years designed to assess the clinical course, prognosis, and risk factors of both AMD and cataracts [24]. In addition to the 
clinical course, AREDS was also designed to evaluate the potential safety and efficacy of antioxidant vitamins and zinc in reducing the incidence or slowing the progression of AMD and/ or cataracts [24]. The initial AREDS study demonstrated that the combination of oral supplements, consisting of the vitamins $\mathrm{C}, \mathrm{E}$, and $\beta$-carotene and minerals, i.e., zinc with copper, reduced the 5-year risk of developing advanced AMD in eyes with intermediate AMD by $25 \%$ and the risk of moderate vision loss by 19\%. The AREDS formulation had no effect on the development or progression of cataract [28].

Data from AREDS, other epidemiologic studies, and animal studies provide a rationale to evaluate the potential impact of other nutrients on the treatment of AMD. Results from observational studies suggest that higher intake of lutein plus zeaxanthin $(\mathrm{L}+\mathrm{Z})$ may reduce the risk of cataract development and progression to advanced AMD [28].

Epidemiologic studies suggest that increased dietary intake of n-3 long-chain polyunsaturated fatty acids (LCPUFAs) and fish products may reduce the risk and incidence of advanced AMD [28-32]. A multicentered follow-up study, the Age-Related Eye Disease Study 2 (AREDS2), sponsored by the NEI, was aimed at evaluating the efficacy of $\mathrm{L}+\mathrm{Z}$ in place of $\beta$-carotene and/or n-3 LCPUFA supplementation adding to the original AREDS formula in reducing the risk of developing advanced AMD [28]. Based on the outcome of the study, addition of either $\mathrm{L}+\mathrm{Z}$, or DHA + EPA, or both to the AREDS formulation did not further reduce the progression to advanced AMD in the primary analysis [33]. After having further examined the data with a secondary analysis, it was concluded that $\mathrm{L}+\mathrm{Z}$ could further reduce the risk of AMD progression and could be more appropriate than $\beta$-carotene in the AREDS formula to reduce the potential risk of lung cancer in smokers [34, 35].

In another clinical study, the LUTEGA study, a combination of $\mathrm{L}+\mathrm{Z}$, antioxidants, and fish oil supplementation for 1 year resulted in a significant increase in macular pigment optical density in nonexudative AMD patients' eyes [36]. L and Z, which are also antioxidants, are the key components of macular pigment. The antioxidant effects of $\mathrm{L}$ and $\mathrm{Z}$ can prevent lipid peroxidation by quenching free radicals or reactive oxygen species [36, 37]. Furthermore, macular pigment absorbs short wavelength with a maximum of $460 \mathrm{~nm}$, acts as an optical filter, and reduces chromatic aberration of the eyes [38]. These results support the use of an $\mathrm{L}+\mathrm{Z}$ supplement in AMD patients to reduce the risk of AMD progression. However, the absence of a significant treatment effect for DHA + EPA in the AREDS2 study merits further discussion.

DHA is the major structural n-3 LCPUFA of retinal photoreceptor outer segment membranes. Biophysical and biochemical properties of DHA may affect photoreceptor membrane function by altering permeability, fluidity, thickness, and lipid phase properties [39]. Tissue DHA status affects retinal cell signaling mechanisms involved in phototransduction. DHA, its precursor EPA, and their metabolites have the capacity to help regulate gene expression, cell signaling, and survival pathways, as well as modulate immune and inflammatory processes implicated in the pathogenesis of retinal vascular and neural cell disease $[28,39]$. In addition to the modulation of inflammatory eicosanoids and mediators, EPA could also directly downregulate VEGF signaling [39] and suppress the CNV induced by laser photocoagulation in animals fed an EPA-rich diet [40]. Dietary n-3 fatty acids (EPA + DHA) also protected animals from hyperoxia-/hypoxia-induced pathological retinal angiogenesis by producing pro-resolving SPMs (NPD1, ResD1, and ResE1) in the retina against neovascularization and suppressed the key proinflammatory cytokine TNF- $\alpha$ [41]. TNF- $\alpha$ plays a crucial role in this disease process; it was shown to upregulate VEGF in retina and retinal pigment epithelium cells and induced an inflammatory process that results in the alteration of the retinal vasculature [42]. Indeed, a pilot clinical study showed that supplementing n-3 fatty acids (1,052 mg fish oil containing $400 \mathrm{mg}$ EPA + $200 \mathrm{mg}$ DHA) daily for 3 months in AMD patients who underwent anti-VEGF intravitreal injection significantly lowered vitreal VEGF 
levels when compared with patients not taking fish oil [43]. This result further strengthens the beneficial effects of $\mathrm{n}-3$ in the treatment of wet AMD.

Against this background of in vitro, in vivo, and clinical studies plus all the observational and epidemiological studies, AREDS2 did not find that taking $n-3$ fatty acids had a significant effect in reducing the progression of AMD. Due to the complex multifactorial nature of the pathological process of AMD, nutritional supplementation usually requires multicomponents for various targets as shown by the success of the first AREDS formulation. Similarly, a recent clinical study using a multicomponent nutritional supplement for Diabetes Visual Function (DiVFuSS) showed meaningful improvement in visual function for retinopathy in diabetic patients [44]. However, based on the review by Souied et al. [45], the design, setting, intake, or subjects of AREDS2 may not have permitted the prophylactic potential of n-3 to be adequately demonstrated. As mentioned in the review, the study design of AREDS2 did not try to investigate the efficacy of $n-3$ fatty acids alone but to identify if the addition can further reduce the risk of AMD by $25 \%$ or more. AREDS2 added the $n-3$ fatty acid supplement to the AREDS1 formula and compared the progression of AMD with patients taking AREDS1 without a real control group with placebo $[33,45]$. It was considered ambitious to achieve a further $25 \%$ risk reduction given that all participants in AREDS2 received the AREDS formulation as a background treatment [45]. Also, as pointed out by Souied et al. [45], subjects in AREDS2 were well educated, predominantly white, well nourished, and likely to be health conscious, with more than $40 \%$ taking cholesterol-lowering drugs and less than $7 \%$ being smokers (compared with the US average at that time of about 19\%). About $20 \%$ of the participants in AREDS2 were $\geq 80$ years old compared to the first AREDS study, where patients were all $<80$ years old at the start of the study [24,28]. Moreover, more than $10 \%$ of the control group subjects took nutritional supplements contravening the protocol guidelines [33,45]. Indeed, a number of key baseline nutritional parameters, including serum L, Z, and DHA/EPA, were significantly better in the AREDS subjects than in the US population as a whole. And specifically, more than $10 \%$ of participants in the control group added a DHA/EPA supplement to their diet $[33,45]$. Therefore, with these confounding factors and limitations in AREDS2, the study was not able to demonstrate the efficacy of n-3 fatty acids in the prevention against AMD.

The only other large prospective randomized clinical study to investigate the prophylactic effects of n-3 on AMD was the Nutritional AMD Treatment 2 (NAT-2) study carried out in France. The NAT-2 was a double-blind, prospective, single-center, randomized, placebocontrolled, comparative trial in 300 patients with neovascular AMD in one eye receiving daily oral n-3 fatty acid capsules (840 mg DHA + $270 \mathrm{mg}$ EPA) or placebo (olive oil capsules) over 3 years, for which the assessment of time to occurrence of CNV in the eye without CNV at entry was the primary efficacy end point. The aim was to evaluate the efficacy of oral PUFA supplementation enriched by DHA in the progression of AMD in a 3-year period [46]. Since the results of their prior pilot study (NAT-1), in which EPA (720 mg) + DHA (480 mg) daily was used for 6 months, showed only a mild DHA increase in patients' blood, it was decided to increase the DHA dose in the NAT-2 study to over $700 \mathrm{mg}$ /day [47]. An important feature of the NAT-2 study was that patients' blood was obtained and levels of n-3 fatty acid in both serum and red blood cell (RBC) membranes were measured as biomarkers that allowed the investigators to evaluate the true compliance objectively and to correlate the $n-3$ fatty acid levels with the risk of AMD. Using RBCs is more reliable than plasma in evaluating $\mathrm{n}-3$ fatty acid status, since clinical studies have shown that levels in RBCs are biologically less variable and a better biomarker for estimated chronic n-3 fatty acid status [48, 49]. Lipid levels in RBCs were shown to correlate very well with lipid levels in retina [50].

Similar to AREDS2 and many clinical studies, compliance to the protocol was an issue that could confound the results and reduce the power to detect a significant difference between 
the placebo and the treatment groups. In the NAT-2 study, it was detected that there was an increase in EPA levels in the placebo group, which may indicate that a significant portion of the patients might have increased their seafood or n-3 supplement intake after study enrollment. Also, about 25\% of the patients in the treatment group had low DHA levels suggesting poor compliance or not responding to supplementation [46]. Therefore, it was not a surprise that the primary outcome analysis of the study did not show any significant treatment effect for a reduction of the incidence of CNV. However, when using the RBC membrane $\mathrm{n}-3$ levels in the secondary analysis, the results showed a significantly lower risk $(-68 \%, p=0.047$; hazard ratio $0.32 ; 95 \%$ confidence interval [CI] 0.10-0.99) of CNV in patients in the n-3 treatment group with a steadily high EPA plus DHA index over 3 years [46]. A subsequent study and analysis of the NAT-2 data more strongly highlighted the relationship between PUFAs and AMD incidence. Patients from the NAT-2 study with neovascular AMD in one eye and early lesions in the other eye were compared with 144 normal controls without AMD [51]. Not only was dietary oily fish and seafood consumption significantly lower in patients with AMD than in controls, but RBC EPA and EPA + DHA were associated with a substantially and significantly lower risk of neovascular AMD (odds ratio $0.25,95 \%$ CI 0.13 $0.47, p<0.0001$, and odds ratio $0.52,95 \%$ CI $0.29-0.94, p=0.03$, respectively) $[45,51]$. A recent study examined 2 independent cohorts of donor eyes from AMD patients and control subjects and related their retinal lipid profiles with systemic biomarkers of lipid intake [50]. They found that RBC lipids correlated very well with retinal lipid in human eyes and are indeed excellent biomarkers of retinal lipid content. Eyes from AMD donors had significantly decreased levels of very long-chain PUFAs and low $n-3 / n-6$ ratios [50], which was consistent with a study showing that AMD patients had a low serum DHA/ARA ratio compared to healthy individuals [52]. All these results support the notion from the NAT-2 study that maintaining a high EPA plus DHA index (Omega-3 Index, 03I) is important in reducing the risk of neovascular AMD.

The 03I (content of EPA + DHA in RBC membranes expressed as percentage of total fatty acid) was developed as a biomarker of tissue n-3 fatty acid status and has been used to evaluate the risk of cardiovascular diseases [53-55]. An 03I of $\geq 8 \%$ has been recommended as a cardioprotective level with the lowest risk of various cardiovascular diseases, and an 03I of $<4 \%$ was associated with the highest risk [55]. In the NAT-2 study, the AMD patients in the n-3 fatty acid treatment group had a baseline 03I average of $3.68 \%$ at the start of the trial. Those patients with an 03I that reached the third tertile (8.68\%) had a reduced risk compared to those with an 03I in the first tertile (4.81\%), with a CNV incidence of $14.3 \%$ compared to $32.5 \%$, respectively, after 3 years [46]. The same recommended cardioprotective 03I level of $\geq 8 \%$ appears to be applicable to reducing the risk in neovascular AMD. It is estimated that an average healthy adult with a low 03I (4.3\%) would require at least $1 \mathrm{~g} /$ day of EPA + DHA for 5 months to achieve an 03I of 8\% [56]. This is the same dosage as recommended by the American Heart Association (AHA) to reduce the risk of death from cardiovascular disease in the secondary prevention setting [57], and it is very close to the dosage (1.1 g) that was used in the NAT- 2 study. Therefore, the $1 \mathrm{~g} /$ day of $\mathrm{n}-3$ fatty acids recommended by the AHA also appears to be appropriate for reducing the risk of neovascular AMD.

\section{Conclusions}

Inflammation plays a significant role in the disease process of DED and AMD. Lipid mediators, including eicosanoids and SPMs derived from membrane fatty acids, are the key players in the inflammatory process and its resolution. In addition to the well-established beneficial effects on reducing the risk of cardiovascular diseases, $n-3$ fatty acids (EPA and DHA) have 
now emerged as being important substrates for the production of SPMs that can bring on the ultimate resolution of the inflammatory process and may modulate angiogenesis, which is crucial in both corneal and choroidal neovascularization, the pathological process involved in certain forms of dry eye and AMD, respectively. Even though EPA and DHA are not considered to be "essential" fatty acids, due to the potential but inefficient conversion of $\alpha$-linolenic acid to EPA and DHA in the human body, the only way to get sufficient EPA and DHA is through dietary intake. Based on the 03I, the dosage recommendation for cardioprotective effects of EPA + DHA (1 g/day) can also be applied to reduce the risk of neovascular AMD progression and can benefit DED patients, especially people with low $n-3$ fatty acid status.

\section{Disclosure Statement}

The authors declare no conflicts of interest.

\section{References}

1 Calder PC: Marine omega-3 fatty acids and inflammatory processes: effects, mechanisms and clinical relevance. Biochim Biophys Acta 2015;1851:469-484.

2 Serhan CN, Chiang N, Dalli J: The resolution code of acute inflammation: novel pro-resolving lipid mediators in resolution. Semin Immunol 2015;27:200-215.

3 Lee SA, Kim HJ, Chang KC, Baek JC, Park JK, Shin JK, et al: DHA and EPA down-regulate COX-2 expression through suppression of NF- $\mathrm{KB}$ activity in LPS-treated human umbilical vein endothelial cells. Korean J Physiol Pharmacol 2009;13:301-307.

4 Rees D, Miles EA, Banerjee T, Wells SJ, Roynette CE, Wahle KW, et al: Dose-related effects of eicosapentaenoic acid on innate immune function in healthy humans: a comparison of young and older men. Am J Clin Nutr 2006;83:331-342.

5 Buckley CD, Gilroy DW, Serhan CN: Proresolving lipid mediators and mechanisms in the resolution of acute inflammation. Immunity 2014;40:315-327.

6 Gronert K: Resolution, the grail for healthy ocular inflammation. Exp Eye Res 2010;91:478-485.

7 Levy BD, Clish CB, Schmidt B, Gronert K, Serhan CN: Lipid mediator class switching during acute inflammation: signals in resolution. Nat Immunol 2001;2:612-619.

8 Hom MM, Asbell P, Barry B: Omegas and dry eye: more knowledge, more questions. Optom Vis Sci 2015; 92: 948-956.

9 Research in dry eye: report of the Research Subcommittee of the International Dry Eye WorkShop (2007). Ocul Surf 2007;5:179-193.

10 Messmer EM: The pathophysiology, diagnosis, and treatment of dry eye disease. Dtsch Arztebl Int 2015;112: 71-81, quiz 2.

11 Baudouin C, Messmer EM, Aragona P, Geerling G, Akova YA, Benitez-Del-Castillo J, et al: Revisiting the vicious circle of dry eye disease: a focus on the pathophysiology of meibomian gland dysfunction. Br J Ophthalmol 2016;100:300-306.

12 Olenik A, Jimenez-Alfaro I, Alejandre-Alba N, Mahillo-Fernandez I: A randomized, double-masked study to evaluate the effect of omega-3 fatty acids supplementation in meibomian gland dysfunction. Clin Interv Aging 2013;8:1133-1138.

13 Liu A, Ji J: Omega-3 essential fatty acids therapy for dry eye syndrome: a meta-analysis of randomized controlled studies. Med Sci Monit 2014;20:1583-1589.

14 Bhargava R, Kumar P: Oral omega-3 fatty acid treatment for dry eye in contact lens wearers. Cornea 2015;34: 413-420.

15 Bhargava R, Kumar P, Phogat H, Kaur A, Kumar M: Oral omega-3 fatty acids treatment in computer vision syndrome related dry eye. Cont Lens Anterior Eye 2015;38:206-210.

16 Bhargava R, Kumar P, Arora Y: Short-term omega 3 fatty acids treatment for dry eye in young and middle-aged visual display terminal users. Eye Contact Lens 2016;42:231-236.

17 Bhargava R, Chandra M, Bansal U, Singh D, Ranjan S, Sharma S: A randomized controlled trial of omega 3 fatty acids in rosacea patients with dry eye symptoms. Curr Eye Res 2016;41:1274-1280.

18 Miljanovic B, Trivedi KA, Dana MR, Gilbard JP, Buring JE, Schaumberg DA: Relation between dietary n-3 and n-6 fatty acids and clinically diagnosed dry eye syndrome in women. Am J Clin Nutr 2005;82:887-893. 
19 Cortina MS, He J, Li N, Bazan NG, Bazan HE: Neuroprotectin D1 synthesis and corneal nerve regeneration after experimental surgery and treatment with PEDF plus DHA. Invest Ophthalmol Vis Sci 2010;51:804-810.

20 Kenchegowda S, Bazan HE: Significance of lipid mediators in corneal injury and repair. J Lipid Res 2010;51: 879-891.

21 Kenchegowda S, He J, Bazan HE: Involvement of pigment epithelium-derived factor, docosahexaenoic acid and neuroprotectin D1 in corneal inflammation and nerve integrity after refractive surgery. Prostaglandins Leukot Essent Fatty Acids 2013;88:27-31.

22 Cortina MS, He J, Russ T, Bazan NG, Bazan HE: Neuroprotectin D1 restores corneal nerve integrity and function after damage from experimental surgery. Invest Ophthalmol Vis Sci 2013;54:4109-4116.

23 Lim A, Wenk MR, Tong L: Lipid-based therapy for ocular surface inflammation and disease. Trends Mol Med 2015;21:736-748.

24 AREDS Research Group: The Age-Related Eye Disease Study (AREDS): design implications. AREDS report No. 1. Control Clin Trials 1999;20:573-600.

25 Blasiak J, Petrovski G, Vereb Z, Facsko A, Kaarniranta K: Oxidative stress, hypoxia, and autophagy in the neovascular processes of age-related macular degeneration. Biomed Res Int 2014;2014:768026.

26 Wang H, Hartnett ME: Regulation of signaling events involved in the pathophysiology of neovascular AMD. Mol Vis 2016;22:189-202.

27 Comparison of Age-Related Macular Degeneration Treatments Trials (CATT) Research Group, Maguire MG, Martin DF, Ying G, Jaffe GJ, Daniel E, et al: Five-year outcomes with anti-vascular endothelial growth factor treatment of neovascular age-related macular degeneration: the comparison of age-related macular degeneration treatments trials. Ophthalmology 2016;123:1751-1761.

28 AREDS2 Research Group, Chew EY, Clemons T, SanGiovanni JP, Danis R, Domalpally A, et al: The Age-Related Eye Disease Study 2 (AREDS2): study design and baseline characteristics (AREDS2 report number 1). Ophthalmology 2012;119:2282-2289.

29 SanGiovanni JP, Agron E, Clemons TE, Chew EY: Omega-3 long-chain polyunsaturated fatty acid intake inversely associated with 12-year progression to advanced age-related macular degeneration. Arch Ophthalmol 2009;127:110-112.

30 Sangiovanni JP, Agron E, Meleth AD, Reed GF, Sperduto RD, Clemons TE, et al: $\omega$-3 long-chain polyunsaturated fatty acid intake and 12-y incidence of neovascular age-related macular degeneration and central geographic atrophy: AREDS report 30, a prospective cohort study from the Age-Related Eye Disease Study. Am J Clin Nutr 2009;90:1601-1607.

31 SanGiovanni JP, Chew EY, Agron E, Clemons TE, Ferris FL 3rd, Gensler G, et al: The relationship of dietary omega-3 long-chain polyunsaturated fatty acid intake with incident age-related macular degeneration: AREDS report No. 23. Arch Ophthalmol 2008;126:1274-1279.

32 SanGiovanni JP, Chew EY, Clemons TE, Davis MD, Ferris FL 3rd, Gensler GR, et al: The relationship of dietary lipid intake and age-related macular degeneration in a case-control study: AREDS report No. 20. Arch Ophthalmol 2007;125:671-679.

33 AREDS2 Research Group: Lutein + zeaxanthin and omega-3 fatty acids for age-related macular degeneration: the Age-Related Eye Disease Study 2 (AREDS2) randomized clinical trial. JAMA 2013;309:2005-2015.

34 Aronow ME, Chew EY: Age-Related Eye Disease Study 2: perspectives, recommendations, and unanswered questions. Curr Opin Ophthalmol 2014;25:186-190.

35 AREDS2 Research Group, Chew EY, Clemons TE, Sangiovanni JP, Danis RP, Ferris FL 3rd, et al: Secondary analyses of the effects of lutein/zeaxanthin on age-related macular degeneration progression: AREDS2 report No. 3. JAMA Ophthalmol 2014;132:142-149.

36 Dawczynski J, Jentsch S, Schweitzer D, Hammer M, Lang GE, Strobel J: Long term effects of lutein, zeaxanthin and omega-3-LCPUFAs supplementation on optical density of macular pigment in AMD patients: the LUTEGA study. Graefes Arch Clin Exp Ophthalmol 2013;251:2711-2723.

37 Xue C, Rosen R, Jordan A, Hu DN: Management of ocular diseases using lutein and zeaxanthin: what have we learned from experimental animal studies? J Ophthalmol 2015;2015:523027.

38 Snodderly DM, Brown PK, Delori FC, Auran JD: The macular pigment. I. Absorbance spectra, localization, and discrimination from other yellow pigments in primate retinas. Invest Ophthalmol Vis Sci 1984;25:660-673.

39 SanGiovanni JP, Chew EY: The role of omega-3 long-chain polyunsaturated fatty acids in health and disease of the retina. Prog Retin Eye Res 2005;24:87-138.

40 Koto T, Nagai N, Mochimaru H, Kurihara T, Izumi-Nagai K, Satofuka S, et al: Eicosapentaenoic acid is antiinflammatory in preventing choroidal neovascularization in mice. Invest Ophthalmol Vis Sci 2007;48:43284334.

41 Connor KM, SanGiovanni JP, Lofqvist C, Aderman CM, Chen J, Higuchi A, et al: Increased dietary intake of omega-3-polyunsaturated fatty acids reduces pathological retinal angiogenesis. Nat Med 2007;13:868-873.

42 Robinson R, Ho CE, Tan QS, Luu CD, Moe KT, Cheung CY, et al: Fluvastatin downregulates VEGF-A expression in TNF- $\alpha$-induced retinal vessel tortuosity. Invest Ophthalmol Vis Sci 2011;52:7423-7431.

43 Rezende FA, Lapalme E, Qian CX, Smith LE, SanGiovanni JP, Sapieha P: Omega-3 supplementation combined with anti-vascular endothelial growth factor lowers vitreal levels of vascular endothelial growth factor in wet age-related macular degeneration. Am J Ophthalmol 2014;158:1071-1078.

44 Chous AP, Richer SP, Gerson JD, Kowluru RA: The Diabetes Visual Function Supplement Study (DiVFuSS). Br J Ophthalmol 2016;100:227-234. 
45 Souied EH, Aslam T, Garcia-Layana A, Holz FG, Leys A, Silva R, et al: Omega-3 fatty acids and age-related macular degeneration. Ophthalmic Res 2015;55:62-69.

46 Souied EH, Delcourt C, Querques G, Bassols A, Merle B, Zourdani A, et al: Oral docosahexaenoic acid in the prevention of exudative age-related macular degeneration: the Nutritional AMD Treatment 2 study. Ophthalmology 2013;120:1619-1631.

47 Querques G, Benlian P, Chanu B, Portal C, Coscas G, Soubrane G, et al: Nutritional AMD treatment phase I (NAT-1): feasibility of oral DHA supplementation in age-related macular degeneration. Eur J Ophthalmol 2009;19:100-106.

48 Harris WS, Thomas RM: Biological variability of blood omega-3 biomarkers. Clin Biochem 2010;43:338-340.

49 Harris WS, Varvel SA, Pottala JV, Warnick GR, McConnell JP: Comparative effects of an acute dose of fish oil on omega-3 fatty acid levels in red blood cells versus plasma: implications for clinical utility. J Clin Lipidol 2013; 7:433-440.

50 Gorusupudi A, Liu A, Hageman GS, Bernstein PS: Associations of human retinal very long-chain polyunsaturated fatty acids with dietary lipid biomarkers. J Lipid Res 2016;57:499-508.

51 Merle BM, Benlian P, Puche N, Bassols A, Delcourt C, Souied EH, et al: Circulating omega-3 fatty acids and neovascular age-related macular degeneration. Invest Ophthalmol Vis Sci 2014;55:2010-2019.

52 Orban T, Johnson WM, Dong Z, Maeda T, Maeda A, Sakai T, et al: Serum levels of lipid metabolites in age-related macular degeneration. FASEB J 2015;29:4579-4588.

53 Harris WS: The Omega-3 Index as a risk factor for coronary heart disease. Am J Clin Nutr 2008;87:1997S2002S.

54 Harris WS: The Omega-3 Index: clinical utility for therapeutic intervention. Curr Cardiol Rep 2010;12:503508.

55 Harris WS, Von Schacky C: The Omega-3 Index: a new risk factor for death from coronary heart disease? Prev Med 2004;39:212-220.

56 Flock MR, Skulas-Ray AC, Harris WS, Etherton TD, Fleming JA, Kris-Etherton PM: Determinants of erythrocyte omega-3 fatty acid content in response to fish oil supplementation: a dose-response randomized controlled trial. J Am Heart Assoc 2013;2:e000513.

57 Kris-Etherton PM, Harris WS, Appel LJ; American Heart Association, Nutrition Committee: Fish consumption, fish oil, omega-3 fatty acids, and cardiovascular disease. Circulation 2002;106:2747-2757.

58 Bhargava R, Kumar P, Kumar M, Mehra N, Mishra A: A randomized controlled trial of omega-3 fatty acids in dry eye syndrome. Int J Ophthalmol 2013;6:811-816.

59 Kangari H, Eftekhari MH, Sardari S, Hashemi H, Salamzadeh J, Ghassemi-Broumand M, et al: Short-term consumption of oral omega-3 and dry eye syndrome. Ophthalmology 2013;120:2191-2196.

60 Kawakita T, Kawabata F, Tsuji T, Kawashima M, Shimmura S, Tsubota K: Effects of dietary supplementation with fish oil on dry eye syndrome subjects: randomized controlled trial. Biomed Res 2013;34:215-220.

61 Epitropoulos AT, Donnenfeld ED, Shah ZA, Holland EJ, Gross M, Faulkner WJ, et al: Effect of oral re-esterified omega-3 nutritional supplementation on dry eyes. Cornea 2016;35:1185-1191. 